\title{
Avaliação de Metas Terapêuticas: Uma Proposta de Arquitetura Direcionada à UbiComp
}

\author{
Alexandre Souza ${ }^{1}$, João Lopes ${ }^{2}$, Leonardo João ${ }^{1}$, Patrícia Davet ${ }^{1}$, \\ Rodrigo Souza $^{2}$, Ana Pernas ${ }^{1}$, Adenauer Yamin ${ }^{1}$, Cláudio Geyer ${ }^{2}$ \\ ${ }^{1}$ Universidade Federal de Pelotas (UFPel) \\ ${ }^{2}$ Universidade Federal do Rio Grande do Sul (UFRGS) \\ \{arrdsouza, ldrsjoao, ptdavet, marilza, adenauer\}@inf.ufpel.edu.br \\ \{jlblopes, rssouza, geyer\}@inf.ufrgs.br
}

\begin{abstract}
This paper proposes a software architecture targeted at Ubiquitous Computing (UbiComp) for providing awareness of the current situation of patients in hospitals. As a central contribution, this work provides the monitoring of the patient's vital signs, allowing the physician to confirm that it is being reached desired effect with the administration of medications. In the developed architecture, this effect desired by physicians in vital parameters is named Therapeutic Goal. The evaluation of the architecture was done through a case study, higlighting the technologies used, and the prototypes developed.
\end{abstract}

Resumo. Este artigo propõe uma arquitetura de software direcionada à Computação Ubíqua (UbiComp) para prover consciência da situação atual de pacientes em ambiente hospitalar. Como contribuição central, este trabalho provê o acompanhamento da evolução dos sinais vitais dos pacientes, permitindo que o médico verifique se está sendo atingido o efeito desejado com a administração dos medicamentos. Na perspectiva da arquitetura desenvolvida, esse efeito procurado pelos médicos nos parâmetros vitais é denominado de Meta Terapêutica. A avaliação da arquitetura foi feita através de um estudo de caso, destacando as tecnologias empregadas e os protótipos desenvolvidos.

\section{Introdução}

O suporte para obtenção da situação dos pacientes é baseado na percepção de sinais vitais dos mesmos, a qual é provida por meio de dados sensoriados. Os sensores coletam dados de contexto dos pacientes, provendo suporte para que o sistema de Computação Ubíqua possa fornecer serviços que reajam de acordo com o contexto. Nesse sentido, a consciência de situação é uma forma promissora de ampliar os modelos tradicionais de monitoramento e alerta, e que pode auxiliar a desenvolver aplicações mais robustas e capazes de melhor atender às necessidades da comunidade em um ambiente hospitalar [Ye et al. 2012].

Desta forma, este trabalho propõe a concepção de uma arquitetura de software para um ambiente ubíquo direcionada a prover consciência da situação atual de pacientes. Essa arquitetura, denominada EXEHDA-TG, acompanha a evolução dos sinais vitais, permitindo que o médico possa confirmar se está sendo atingido o efeito desejado com a administração dos medicamentos prescritos. Esse comportamento desejado pelos médicos nos parâmetros vitais é, na arquitetura proposta, chamado de Meta Terapêutica. 
Como contribuição central, a arquitetura desenvolvida automatiza a aquisição e viabiliza a avaliação dos sinais vitais com base no conhecimento dos médicos, os quais especificam a Meta Terapêutica a ser perseguida. O EXEHDA-TG possibilita o envio de alertas aos profissionais de saúde em uma perspectiva ubíqua, provendo mobilidade no acesso às informações médicas.

A arquitetura proposta tem como premissa sua integração ao middleware EXEHDA (Execution Environment for Highly Distributed Applications). O EXEHDA provê uma arquitetura de software que visa criar e gerenciar um ambiente ubíquo, bem como promover a execução, sob este ambiente, das aplicações da computação ubíqua [Lopes et al. 2014].

O artigo está organizado da seguinte forma: a seção 2 descreve as funcionalidades da arquitetura proposta. A seção 3 apresenta um estudo de caso. A seção 4 discute os trabalhos relacionados. Por fim, a seção 5 apresenta as considerações finais.

\section{EXEHDA-TG: Arquitetura e Funcionalidades}

O EXEHDA-TG mantém a proposta organizacional do middleware EXEHDA, sendo composto de dois tipos principais de servidores: (i) Servidor de Integração: responsável pela relação com o meio através de sensores e atuadores; e (ii) Servidor de Avaliação: responsável pelo armazenamento e processamento das informações contextuais referentes aos pacientes, coletadas do ambiente ubíquo provido pelo EXEHDA.

O ambiente ubíquo gerenciado pelo EXEHDA tem uma organização celular, onde cada célula pode empregar vários Servidores de Integração, sendo que cada um provê dados a um Servidor de Avaliação, e pode coletar dados de vários leitos. Uma célula pode atender um ou mais hospitais e, por sua vez, um hospital de grande porte pode ter mais de uma célula.

O EXEHDA-TG tem como objetivo fornecer uma solução capaz de: (i) coletar de forma automatizada informações contextuais dentro do ambiente hospitalar; (ii) processar estas informações contextuais coletadas; e (iii) prover alternativas de atuação sobre o meio e permitir o envio de alertas a dispositivos móveis.

A descrição do EXEHDA-TG, feita a seguir, está organizada a partir de seus servidores e respectivas funcionalidades, sendo realizadas as necessárias associações entre os mesmos, e com os outros serviços do middleware EXEHDA.

\subsection{Servidor de Integração}

O Servidor de Integração foi concebido para prover as funcionalidades necessárias para interfaceamento com os equipamentos eletromédicos, viabilizando no meio ubíquo a aquisição de dados através de sensores, bem como os pertinentes acionamentos de atuadores. Na Figura 1 é apresentada a arquitetura desse servidor.

O Módulo de Aquisição é responsável pela coleta e tratamento dos dados de sensoriamento das bombas de infusão e monitores de sinais vitais. Este módulo realiza a interface para captura das informações dos sensores dos equipamentos eletromédicos, considerando os protocolos de comunicação necessários para cada tipo de dispositivo. A leitura dos sensores é realizada de acordo com o agendamento definido pelas Metas Terapêuticas. Os dados coletados são tratados em etapas, através das quais é feita sua 


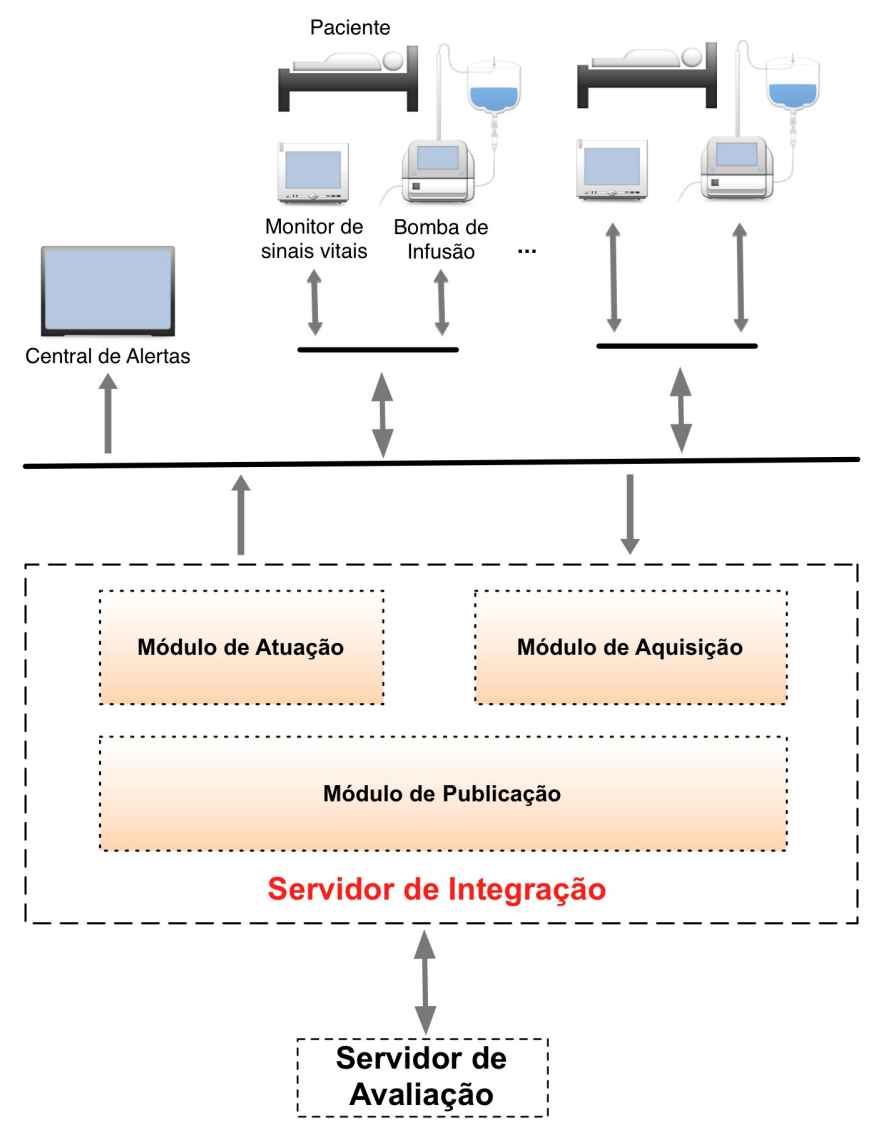

Figura 1. Servidor de Integração do EXEHDA-TG

normalização e a definição de quais dados devem ser efetivamente publicados no Servidor de Avaliação.

O Módulo de Publicação é responsável por coordenar o principal fluxo de dados entre os Servidores de Integração e o Servidor de Avaliação, promovendo a publicação dos dados coletados e garantindo uma persistência local dos mesmos quando a comunicação entre estes servidores estiver temporariamente indisponível.

O Módulo de Atuação tem como função acionar os dispositivos dos equipamentos eletromédicos responsáveis por apresentar alertas visuais e sonoros aos profissionais de saúde, tais como: displays de cristal líquido (LCD - Liquid Crystal Display), buzzers, altofalantes, LEDs (Light-Emitting Diode), etc. Portanto, as mensagens apresentadas nas telas dos equipamentos eletromédicos são considerados um tipo específico de atuação.

\subsection{Servidor de Avaliação}

O Servidor de Avaliação foi desenvolvido para ser o responsável por receber valores de contexto coletados dos pacientes e dos equipamentos eletromédicos, armazenar os mesmos e disparar avaliações situacionais solicitando atuações e alertas sempre que necessário. A Figura 2 mostra uma visão geral desse servidor, onde é apresentada a sua relação com os Servidores de Integração, aplicações de administradores, aplicações ubíquas dos profissionais de saúde, outros servidores de avaliação e serviços administrativos do hospital. 


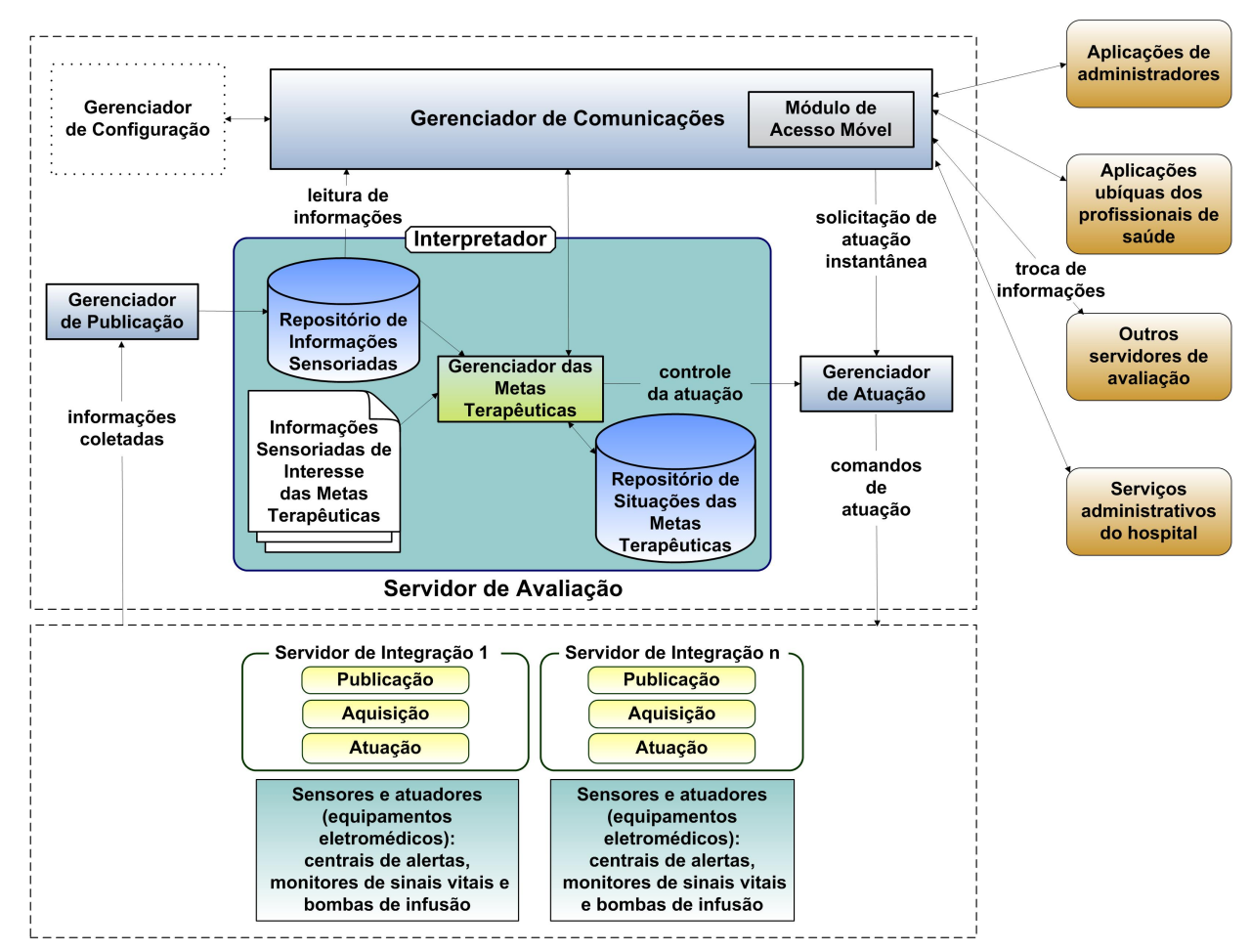

Figura 2. Servidor de Avaliação do EXEHDA-TG

Os módulos que compõem o Servidor de Avaliação são responsáveis pela consciência de situação, desde a aquisição de dados contextuais até o acionamento de atuadores e o envio de alertas. A seguir está a descrição destes módulos.

O Gerenciador de Configuração, através das aplicações de administradores, provê as funcionalidades necessárias para realizar a parametrização e gerenciamento do Servidor de Avaliação, considerando as características dos sensores e atuadores dos equipamentos eletromédicos. Este módulo armazena, em um repositório próprio, a programação das bombas de infusão, definições das Meta Terapêuticas e os parâmetros de aquisição para os dispositivos eletromédicos.

O Gerenciador de Comunicações é composto por um barramento ESB (Enterprise Service Bus) e um Módulo de Acesso Móvel, os quais possuem as funcionalidades necessárias para atendimento das aplicações distribuídas dos usuários. As aplicações ubíquas dos profissionais de saúde e outros Servidores de Avaliação utilizam o Gerenciador de Comunicações para solicitar dados de sinais vitais, status e parâmetros dos equipamentos eletromédicos e para acionar atuadores. Essas aplicações ubíquas dos profissionais de saúde estão em execução nos diferentes nodos que integram a estrutura computacional ubíqua provida pelo middleware EXEHDA.

Com a intenção de prover acesso em deslocamento ao EXEHDA-TG foi concebido o Módulo de Acesso Móvel, o qual está organizado em dois blocos.

Bloco de Exibição de Informações Contextuais: executa no dispositivo móvel e disponibiliza ao usuário relatórios, tanto gráficos como textuais, acerca das informações contextuais de seu interesse. A comunicação do bloco com o Servidor de Avaliação acontece em duas etapas: (i) é feita uma inspeção das informações de contexto que estão sendo 
tratadas; e (ii) são solicitados dados específicos para determinada informação contextual em um intervalo de tempo. As funcionalidades estão organizadas em três módulos: (i) Módulo de Relatório: trata da exibição das informações contextuais no dispositivo móvel; (ii) Módulo de Acesso: consiste de um servidor com total acesso ao Repositório de Informações Sensoriadas; e (iii) Módulo de Requisição: tem por objetivo solicitar informações contextuais ao Módulo de Acesso, a pedido do Módulo de Relatório.

Bloco de Tratamento de Alertas Proativos: tem por objetivo sinalizar o usuário quando da ocorrência de eventos de seu interesse através de notificações proativas. As funcionalidades estão organizadas em dois módulos: (i) Módulo de Alertas, executa no dispositivo móvel, disponibilizando alertas ao usuário, empregando para isso o mecanismo nativo de notificação da plataforma móvel. Esta opção tem como característica propiciar ao usuário um gerenciamento integrado da natureza dos alertas praticados pelo seu dispositivo móvel; e (ii) Módulo de Distribuição, executa no mesmo equipamento do Servidor de Avaliação, em regime de operação ininterrupta. Este módulo opera mantendo os alertas produzidos pelas diferentes regras contextuais, tratadas pelo Interpretador. Seu acesso pelos dispositivos móveis ocorre através do Módulo de Comunicação, o qual, através de um barramento ESB, propicia acesso as diferentes funcionalidades do Servidor de Avaliação.

O Gerenciador de Publicação é responsável pelo suporte à coleta de sinais vitais, status e parâmetros das bombas de infusão e monitores de sinais vitais, capturados através do Servidor de Integração. Essas funcionalidades são implementadas através do barramento ESB do Servidor de Avaliação.

O Gerenciador de Atuação é responsável pelo acionamento dos atuadores dos equipamentos eletromédicos. Esse componente tem como função disparar no ambiente ubíquo ações que mudem o estado dos equipamentos eletromédicos, apresentando alertas visuais e sonoros aos profissionais de saúde, sempre que solicitado pelos demais módulos do Servidor de Avaliação.

O Interpretador tem como principal função realizar as tarefas de gerenciamento e identificação das informações contextuais, utilizando para isto dados especificados no componente Informações Sensoriadas de Interesse das Metas Terapêuticas. A tarefa de identificação é processada sobre os dados coletados através dos sensores e tem o intuito de produzir informações de contexto de mais alto nível. A seguir são apresentados os componentes deste módulo.

Repositório de Informações Sensoriadas: armazena os dados de contexto coletados pelos sensores dos equipamentos eletromédicos. A estrutura prototipada traduz a organização da arquitetura do middleware EXEHDA, contemplando as relações entre as aplicações, componentes, sensores, ambientes e os contextos de interesse. Neste repositório estão armazenados os sinais vitais dos pacientes e os dados de sensoriamento das bombas de infusão e monitores, obtidos através do Módulo de Aquisição do Servidor de Integração, mantendo dessa forma um registro dos contextos que podem ser avaliados pelo Gerenciador da Meta Terapêutica. As informações de contexto capturadas são registradas neste repositório juntamente com seu timestamp da coleta, timestamp de registro e Servidor de Integração de origem. As informações deste repositório podem ser acessadas pelas aplicações ubíquas dos profissionais de saúde através do Gerenciador de 
Comunicações.

Repositório de Situações: armazena os resultados das avaliações realizadas pelo Gerenciador da Meta Terapêutica sobre as informações contextuais oriundas do Repositório de Informações Sensoriadas. Este repositório mantém um histórico de quando e quais Metas Terapêuticas foram atingidas. As informações deste repositório podem ser acessadas pelas aplicações ubíquas dos profissionais de saúde através do Gerenciador de Comunicações.

Gerenciador das Metas Terapêuticas: é um mecanismo de inferência baseado em regras, o qual produz contextos deduzidos de acordo com as Metas Terapêuticas definidas pelos profissionais de saúde. O Gerenciador das Metas Terapêuticas processa regras do tipo Evento-Condição-Ação para identificação de situações, utilizando técnicas baseadas em Lógica Temporal e Espacial [Allen and Ferguson 1994] [Ye et al. 2012]. Os parâmetros destas regras são definidos pelos profissionais de saúde, levando em consideração as faixas de valores e o tempo esperado como meta para os sinais vitais dos pacientes em função da administração de um medicamento. Quando um dado coletado é publicado no Repositório de Informações Sensoriadas, o Gerenciador das Metas Terapêuticas consulta o componente Informações Sensoriadas de Interesse das Metas Terapêuticas para identificar quais regras devem ser avaliadas. Os resultados destas avaliações são então registrados no Repositório de Situações das Metas Terapêuticas.

\section{Estudo de Caso}

Esta seção apresenta as tecnologias utilizadas na concepção do EXEHDA-TG, bem como o estudo dos cenários de uso empregados na avaliação de suas funcionalidades. O estudo de caso desenvolvido contempla tarefas referentes ao sensoriamento e a coleta de informações contextuais, avaliação e notificação dos dados de contexto aos demais serviços do middleware.

A bomba de infusão e o monitor de sinais vitais utilizados neste estudo de caso possuem comunicação de dados via WiFi. Com isso, é possível remotamente parametrizar os dados de uma nova infusão, fazer a leitura dos sinais vitais coletados, verificar o estado dos sensores/alarmes e o andamento da infusão.

Foram utilizadas plataformas computacionais baseadas em Linux para viabilizar, no ambiente ubíquo, a aquisição de dados através de sensores e o acionamento de atuadores. Como Servidor de Integração foi utilizada a Raspberry Pi modelo B (www.raspberrypi.org), cujas características principais são apresentar tamanho reduzido, baixo consumo de energia e custo reduzido, quando comparada com desktop convencionais.

O Servidor de Avaliação é responsável por receber valores de contexto coletados dos pacientes e dos equipamentos eletromédicos, armazenar os mesmos e disparar avaliações situacionais, solicitando atuações e gerando alertas sempre que necessário. Para isto, neste estudo de caso foi utilizado um hardware com processador Intel E34002.6GHz de dois núcleos, com memória RAM de 4Gb, com o Sistema Operacional Ubuntu Server.

Neste estudo de caso foram desenvolvidas duas aplicações com o objetivo de acompanhamento e avaliação das Metas Terapêuticas: a primeira é utilizada com na- 
vegadores Web e a segunda é direcionada para a plataforma Android.

Através da aplicação para navegadores é possível: (i) consultar os dados de contexto coletados pelos sensores dos equipamentos eletromédicos e armazenados no Repositório de Informações Sensoriadas do Servidor de Avaliação, visualizando-os através de gráficos e tabelas; (ii) visualizar relatórios textuais com os dados coletados da última semana; (iii) acompanhar a evolução de mais de um sinal vital em um mesmo gráfico, permitindo compará-los; e (iv) realizar análises estatísticas dos dados coletados.

$\mathrm{Na}$ tela inicial da aplicação para navegadores é possível fazer a seleção de pacientes e os dados clínicos de interesse do profissional de saúde. Na Figura 3 é apresentado um gráfico típico da evolução dos sinais vitais. Através do mesmo é possível acompanhar a variação dos sinais vitais em função da medicação administrada ao paciente. Posicionando o mouse no gráfico é detalhado o valor e o momento (data e horário) de coleta do mesmo. Também é possível visualizar mais de um sinal vital simultaneamente em um mesmo gráfico, sendo viabilizada assim a comparação entre os mesmos.

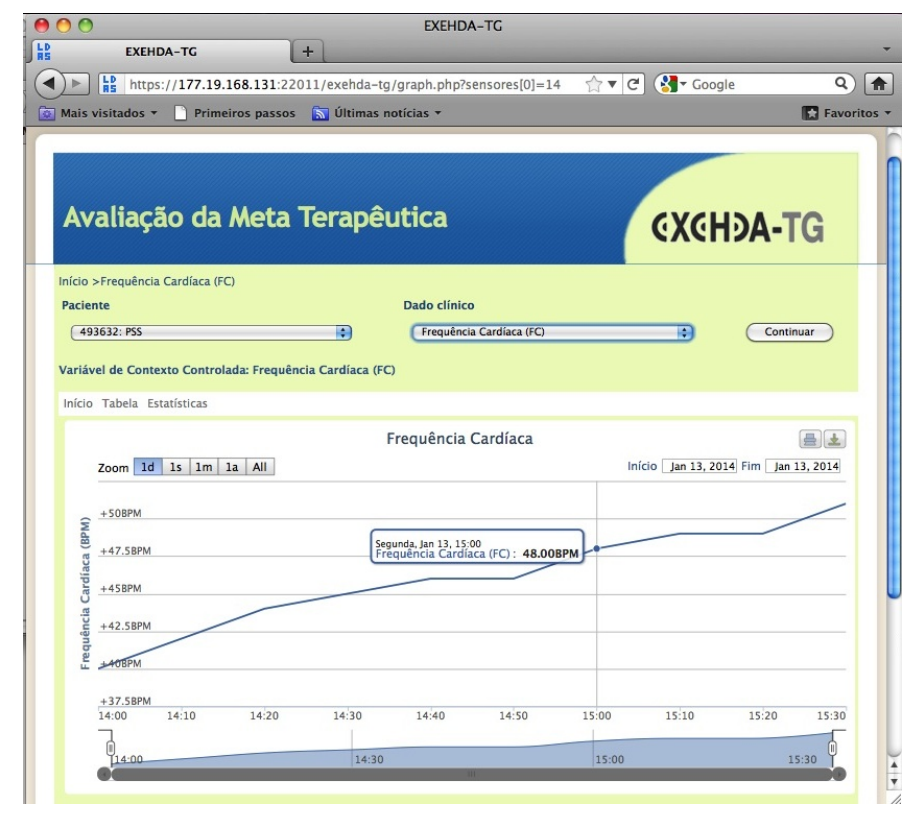

Figura 3. EXEHDA-TG: Evolução dos Sinais Vitais

Através da opção da interface Web do EXEHDA-TG pode-se visualizar os sinais vitais de um paciente coletados na última semana. Estas informações são apresentadas em colunas, uma para cada dia da semana, onde também é possível ver os valores médio, máximo e mínimo de cada dia. Na opção "Estatística" (vide Figura 4) é apresentada uma funcionalidade da aplicação Web que viabiliza o cruzamento de dados contextuais envolvendo múltiplos sinais vitais a partir de diferentes regras. Esse recurso permite a adição, remoção e edição de regras e parâmetros.

A prototipação do Módulo de Acesso Móvel é constituída de duas partes que interoperam: uma aplicação móvel e outra que processa junto ao Servidor de Avaliação. A parte móvel é voltada para smartphones e/ou tablets que empregam o sistema operacional Android. Na tela de abertura da aplicação é possível selecionar um paciente, o dado clínico de interesse e a forma (gráfica ou textual) desejada para visualizar o mesmo. Na 


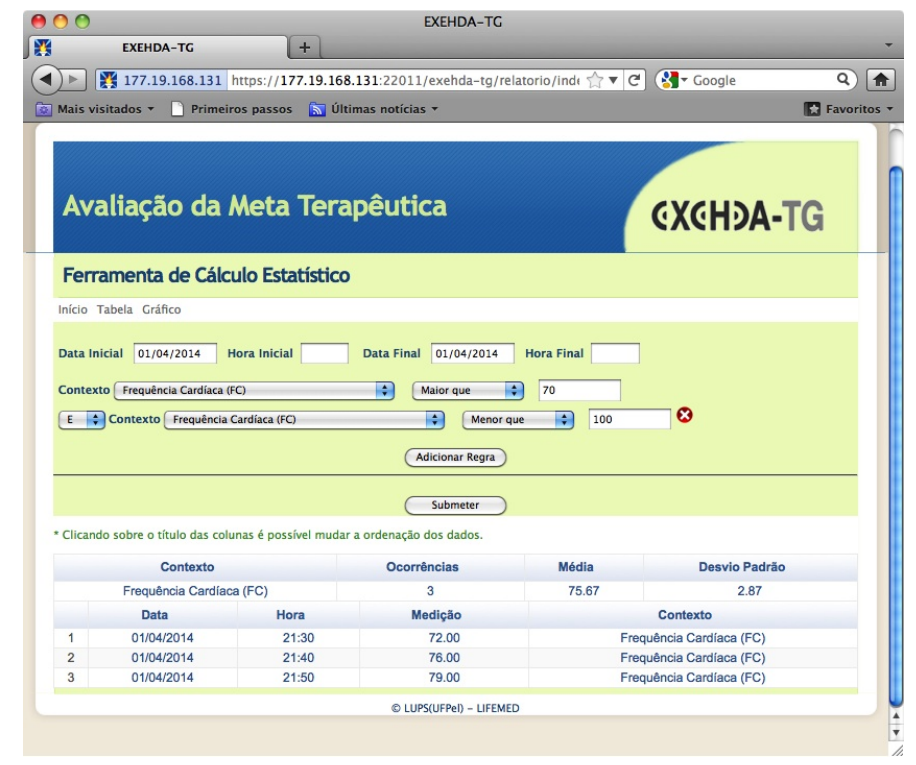

Figura 4. EXEHDA-TG: Informações Estatísticas

tela de informações gráficas da aplicação móvel é apresentado o gráfico com a evolução dos sinais vitais, onde é possível selecionar o período de tempo desejado de exibição (uma hora, um dia, uma semana ou um ano). Ao clicar na curva do sinal vital é apresentado o valor e o momento (data e horário) de coleta do mesmo. O eixo vertical do gráfico é ajustado de forma automática, levando em consideração os valores mínimos e máximos a serem plotados.

Para disponibilização dos alertas ao usuário foi utilizado o mecanismo nativo de notificação da plataforma Android (vide Figura 5). Esta opção tem como característica propiciar ao usuário um gerenciamento integrado da natureza dos alertas praticados pelo seu dispositivo móvel.

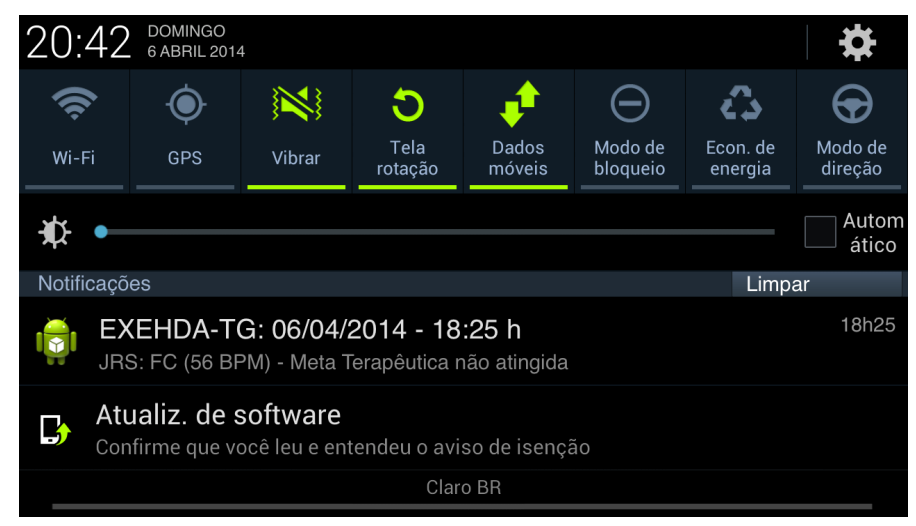

Figura 5. EXEHDA-TG: Envio de Alerta na Aplicação Android

\section{Trabalhos Relacionados}

A discussão dos trabalhos relacionados abrangeu os seguintes projetos: MTMCC [Koutkias et al. 2010], HeartCycle [Chouvarda et al. 2011], SIMAp [Leite 2011] e PA-DSS [Gunawardane et al. 2009]. Esta discussão teve por base aspectos decorrentes 
das premissas consideradas para concepção do EXEHDA-TG: arquitetura distribuída ou centralizada, armazenamento e consulta dos dados de contexto, suporte ao tratamento de regras, regras baseadas no conhecimento de médicos especialistas, verificação da resposta ao tratamento medicamentoso, interface com equipamentos eletromédicos e abordagem siga-me para envio de alertas. Estes aspectos são considerados na comparação com os trabalhos relacionados apresentada na Tabela 1.

Tabela 1. Comparação com os Trabalhos Relacionados

\begin{tabular}{llcccc}
\hline Características & \multicolumn{5}{c}{ Projetos } \\
\hline \hline & MTM-CC & HeartCycle & SIMAp & PA-DSS & EXEHDA-TG \\
\hline $\begin{array}{l}\text { Armazenamento e consulta sobre } \\
\text { os dados de contexto }\end{array}$ & não & $\operatorname{sim}$ & não & não & $\operatorname{sim}$ \\
\hline Suporte ao tratamento de regras & sim & não & sim & sim & $\operatorname{sim}$ \\
\hline $\begin{array}{l}\text { Regras baseadas no conhecimento } \\
\text { de médicos especialistas }\end{array}$ & $\operatorname{sim}$ & - & $\operatorname{sim}$ & $\operatorname{sim}$ & $\operatorname{sim}$ \\
\hline $\begin{array}{l}\text { Verificação da resposta ao tratamento } \\
\text { medicamentoso }\end{array}$ & sim & sim & não & não & $\operatorname{sim}$ \\
\hline $\begin{array}{l}\text { Interface com equipamentos } \\
\text { eletromédicos }\end{array}$ & não & sim & sim & sim & sim \\
\hline $\begin{array}{l}\text { Abordagem siga-me para envio } \\
\text { de alertas }\end{array}$ & não & não & sim & sim & sim \\
\hline
\end{tabular}

Todos os projetos estudados possuem arquitetura distribuída, e de forma análoga ao EXEHDA-TG, contemplam esforços de processamento dos dados contextuais em diferentes posições da arquitetura. O armazenamento dos dados de contexto é uma funcionalidade presente em todos os projetos. Porém, apenas o EXEHDA-TG disponibiliza facilidades para consulta posterior dos dados coletados.

Com exceção do projeto HeartCycle, os demais provêem suporte ao tratamento de regras. O EXEHDA-TG diferencia-se dos trabalhos relacionados por permitir a definição das Metas Terapêuticas de forma personalizada através do uso de regras, viabilizando seu emprego para diversos propósitos. Assim, no EXEHDA-TG a parametrização da Meta Terapêutica é feita pelos médicos , os quais podem criar regras para serem informados, através de alertas, caso os valores dos sinais vitais satisfaçam alguma condição que coloque o paciente em risco ou caso algum sintoma não desejado seja identificado.

Os projetos SIMAp e PA-DSS não consideram a evolução dos sinais vitais em função do tratamento medicamentoso de forma personalizada por paciente. O EXEHDATG diferencia-se por gerar alertas quando os sinais vitais não estão evoluindo em função da medicação, conforme o esperado pelo médico em cada caso clínico.

No projeto MTM-CC os sinais vitais são coletados através de uma rede de sensores biomédicos sem fio. Por sua vez, o EXEHDA-TG, de forma análoga aos projetos HeartCycle, SIMAp e PA-DSS, coleta os sinais vitais dos pacientes através de monitores. Entretanto, o EXEHDA-TG diferencia-se destes trabalhos por também possuir suporte para interface com bombas de infusão, permitindo avaliar e armazenar os alarmes gerados pelas mesmas.

No projeto MTM-CC os alertas são enviados a um servidor central, enquanto no EXEHDA-TG estes alertas são enviados tanto para uma central de alertas no ambiente hospitalar, como para os dispositivos móveis dos profissionais de saúde. Com isso, o EXEHDA-TG diferencia-se por suportar a abordagem siga-me, a qual permite ao usuário 
iniciar aplicações e acessar dados a partir de qualquer lugar e tipo de equipamento, todo o tempo.

\section{Considerações Finais}

A concepção de uma arquitetura para o acompanhamento dos sinais vitais em função da medicação, se mostra uma ferramenta oportuna para avaliar a resposta do paciente ao tratamento, bem como para enviar alertas aos profissionais de saúde quando os valores dos sinais vitais indicarem uma resposta inesperada ao medicamento.

Desta forma, através do recebimento destes alertas, o médico pode reavaliar a prescrição dos medicamentos ou prescrever um novo fármaco para que o tratamento tenha a resposta desejada. Este pronto aviso à equipe médica de que a Meta Terapêutica não foi atingida, constitui uma das contribuições centrais do EXEHDA-TG para agilização das práticas relacionadas ao tratamento de pacientes, com potencial contribuição para que o tempo de internação seja reduzido.

Dentre outros, na continuidade da pesquisa, os seguintes aspectos deverão ser considerados em trabalhos futuros: (i) realizar a validação clínica no ambiente hospitalar, analisando o desempenho do EXEHDA-TG em uma situação cotidiana; (ii) explorar o emprego de regras de processamento contextual que utilizem outros mecanismos de inferência de mais alto nível, ampliando as opções de inferência sobre os dados coletados; e (iii) dar continuidade aos procedimentos de integração do EXEHDA-TG com os diferentes serviços e funcionalidades do middleware EXEHDA.

\section{Referências}

Allen, J. F. and Ferguson, G. (1994). Actions and Eventis in Interval Temporal Logic. Journal of Logic and Computation.

Chouvarda, I., Gkontra, P., Kokonozi, A., Semertzidis, P., Caffarel, J., and Maglaveras, N. (2011). Novel Approaches for Medication Compliance and Effectiveness Analysis and Support in Cardiovascular Disease Patients. In 33rd Annual International Conference of the IEEE EMBS.

Gunawardane, T. S. F. W., Koggalage, R., Rodrigo, R., and Rajapakse, S. (2009). Patient Alert and Decision Support System. In ACTEA 2009: 2009 International Conference on Advances in Computational Tools for Engineering Applications.

Koutkias, V. G., Chouvarda, I., Triantafyllidis, A., Malousi, A., Giaglis, G. D., and Maglaveras, N. (2010). A Personalized Framework for Medication Treatment Management in Chronic Care. IEEE Transactions On Information Technology In Biomedicine.

Leite, C. R. M. (2011). Arquitetura Inteligente Fuzzy para Monitoramento de Sinais Vitais de Pacientes: Um Estudo de Caso em UTI. PhD thesis, Universidade Federal do Rio Grande do Norte - Programa De Pós-Graduação em Engenharia Elétrica e Computação.

Lopes, J., Souza, R., Geyer, C., Costa, C., Barbosa, J., Pernas, A., and Yamin, A. (2014). A middleware architecture for dynamic adaptation in ubiquitous computing. Journal of Universal Computer Science, 20(9):1327-1351.

Ye, J., Dobson, S., and McKeever, S. (2012). Situation identification techniques in pervasive computing: A review. Pervasive and Mobile Computing. 